\title{
A New Method of Moments for the Bimodal Particle System in the Stokes Regime
}

\author{
Yan-hua Liu ${ }^{1}$ and Zhao-qin Yin ${ }^{2}$ \\ ${ }^{1}$ College of Mechanical and Electrical Engineering, Hohai University, Changzhou 213022, China \\ ${ }^{2}$ China Jiliang University, Hangzhou 310018, China \\ Correspondence should be addressed to Yan-hua Liu; liuyanhua@zju.edu.cn
}

Received 22 September 2013; Accepted 30 October 2013

Academic Editor: Jianzhong Lin

Copyright (c) 2013 Y.-h. Liu and Z.-q. Yin. This is an open access article distributed under the Creative Commons Attribution License, which permits unrestricted use, distribution, and reproduction in any medium, provided the original work is properly cited.

\begin{abstract}
The current paper studied the particle system in the Stokes regime with a bimodal distribution. In such a system, the particles tend to congregate around two major sizes. In order to investigate this system, the conventional method of moments (MOM) should be extended to include the interaction between different particle clusters. The closure problem for MOM arises and can be solved by a multipoint Taylor-expansion technique. The exact expression is deduced to include the size effect between different particle clusters. The collision effects between different modals could also be modeled. The new model was simply tested and proved to be effective to treat the bimodal system. The results showed that, for single-modal particle system, the results from new model were the same as those from TEMOM. However, for the bimodal particle system, there was a distinct difference between the two models, especially for the zero-order moment. The current model generated fewer particles than TEMOM. The maximum deviation reached about $15 \%$ for $m_{0}$ and $4 \%$ for $m_{2}$. The detailed distribution of each submodal could also be investigated through current model.
\end{abstract}

\section{Introduction}

The particulate matter has become one of the most dangerous pollutants to the atmospheric environment and the health of human beings. It will reduce the visibility of the atmosphere and cause the traffic crowding and serious accidents. The fine particles (PM2.5) will also be breathed into the bronchus of human beings, followed by several kinds of respiratory diseases. The lungs will absorb the fine particles and cardiovascular disease will come into being [1]. However, the mechanism of the generation and evolution of the particulate matter still remains to be clarified. Hence, it has both theoretical and realistic senses to study the dynamics of the particulate matter.

Previous study on the aerosol dynamics usually supposes that the particle system is monodispersed (i.e., the system has only one scale) or multidispersed (i.e., the system has multiscales) but is in a log-normal distribution in size [2]. Such kinds of assumptions will greatly simplify the problems, and a series of approximate or precise solutions will be obtained. However, these assumptions are based on the experimental measurement and cannot be applied to all the cases. There is another type of particle size distribution, namely, bimodal or multimodal distribution. For example, the newborn particles together with the background particles compose the bimodal distribution system. Furthermore, the newborn particles may also exhibit a multimodal or bimodal distribution [3]. Pugatshova et al. [4] and Lonati et al. [5] measured the particulate matter in the urban on-road atmosphere in different cities and times. The multimodal distribution was observed. At this time, unacceptable error may appear using mono-dispersed or log-normal assumption.

Take the bimodal system, for example: the particles gather around two independent particle sizes. In order to study such a system, the particle size distribution should be separated into two sub-PSDs [6]. The dynamics of the system may be obtained according to the two subparticle clusters. Under this description, the governing equations of the particle system should be modified to represent the additional coagulation effect [7]; that is, the collision of particles is artificially separated into two kinds: internal coagulation and external 
coagulation. Because the typical particle diameter of the bimodal system is $5 \mathrm{~nm}$ to $2.5 \mu \mathrm{m}$, which means that particles lie in different dynamic regimes (free molecular regime, transition regime and continuum regime), the coagulation in such a wide range should also be treated separately. The current study will focus on the continuum (Stokes) regime.

Generally, the particle balance equation (PBE) governs the detailed evolution process of PSD and can be numerically solved. However, because of its huge computation resource to solve the PBE directly, the method of moment (MOM) $[2,8,9]$ is often taken into account as an alternation. It takes several moments of PSD in particle volume space and converts PBE into moment equations. Each moment has its physical meaning: zero-order moment represents the number concentration, first-order moment represents the volume concentration, and second-order moment is related to the polydispersity. Although MOM cannot directly give out the evolution of specific PSD, it can obtain the statistical characteristics of particle system and the calculation during this procedure reduces to an acceptable level. As a matter of fact, MOM is widely used in the research of aerosol dynamics for its simplicity and low computational cost.

One limitation of MOM is the closure problem due to the coagulation term in PBE. When PBE is converted into moment equations, the coagulation term will be transformed into fractional moments, which cannot be explicitly expressed and mathematical models should be introduced into MOM to solve this problem, the so-called closure problem. There are typically three kinds of methods: predetermined PSD [2], quadrature method of moment (QMOM) [10], and Taylor-expansion method of moment (TEMOM) [11]. The first class often supposes that the PSD is a log-normal distribution, and the coagulation term can be directly determined. It can only be applied to the log-normal distributed particle system. QMOM utilizes the Gaussian quadrature method to evaluate the coagulation term in the moment equations. The pre-determined PSD is not necessary, but the computation is easy to diverge. TEMOM expands the nonlinear term in the collision kernel using the Taylor expansion. Finally, the coagulation term can be expressed as a linear combination of different moments. TEMOM has its superiority on its easy expression, high precision, and low computational cost. It is widely used in the research of the aerosol dynamics [12-15].

However, using TEMOM to study the bimodal system has some problems. TEMOM expands the collision kernel function at the average diameter $u_{0}$. For the internal collision, there is no problem, but, for the external collision in bimodal system, this expansion should be extended. For a typical bimodal system, there are two clusters of particles with different diameters, and the total numbers of particles in each cluster are also different. This fact will contribute to the fact that the average diameter of the whole system may lie around the first mode or the second mode or even the mid place of the two modes. If the external collision term also expands at the average diameter of the system, additional error will decrease the accuracy of the simulation. In TEMOM, the convergent region is $\left(0,2 u_{0}\right)[16]$, while, for bimodal system, one mode may lie outside this region if both modes expand at the same point. This possibility may lead to the divergence of the calculation.

Hence, the Taylor-expansion method of moments should be developed to be applied to the bimodal particle system to improve the accuracy and the stability. The current research will focus on the multipoint Taylor-expansion method of moments, and the Stokes regime is preferred for ease.

\section{Mathematical Theories}

Considering the typical system with Brownian coagulation only, PSD satisfies the PBE as [2]

$$
\begin{aligned}
\frac{\partial N(v)}{\partial t}= & \frac{1}{2} \int_{0}^{v} \beta(u, v-u) N(u) N(v-u) d u \\
& -N(v, t) \int_{0}^{\infty} \beta(v, u) N(u) d u,
\end{aligned}
$$

where $N(v)$ is the size distribution function, which means the number of particles with a volume $v, u$ and $v$ are the particle volumes, and $\beta$ is the Brownian coagulation coefficient.

In order to convert PBE into moment equations, the definition of moments is introduced

$$
m_{k}(t)=\int_{0}^{\infty} N(v, t) v^{k} d v a .
$$

Applying (2) to (1), the moment equations are obtained:

$$
\begin{aligned}
\frac{\partial m_{k}}{\partial t}=\frac{1}{2} \iint_{0}^{\infty}\{ & {\left[\left(v_{1}+v_{2}\right)^{k}-v_{1}^{k}-v_{2}^{k}\right] } \\
& \left.\times \beta\left(v_{1}, v_{2}\right) N\left(v_{1}\right) N\left(v_{2}\right)\right\} d v_{1} d v_{2}
\end{aligned}
$$

In current paper, the Stokes regime is studied, and the collision kernel $\beta$ may be rewritten as

$$
\beta_{\mathrm{c}}\left(v_{1}, v_{2}\right)=B\left[2+\left(\frac{v_{2}}{v_{1}}\right)^{1 / 3}+\left(\frac{v_{1}}{v_{2}}\right)^{1 / 3}\right] \text {, }
$$

where $B=2 k_{b} T / \mu, k_{b}$ is the Boltzmann constant, $T$ is the environment temperature, and $\mu$ is the molecular viscosity of gas.

When investigating the bimodal system, PSD can be expressed as $N(v, t)=N_{i}(v, t)+N_{j}(v, t)$. PBE for each subPSD can be established. Apply the definition equation (2) to the PBEs. The moment equations can be attained for both cluster $i$ and cluster $j$ listed as follows:

$$
\begin{aligned}
& \frac{\partial m_{k}^{i}}{\partial t}=C_{k}^{i i}+D_{k}^{i j}, \\
& \frac{\partial m_{k}^{j}}{\partial t}=C_{k}^{j j}+E_{k}^{i j},
\end{aligned}
$$


where

$$
\begin{aligned}
& C_{k}^{i i}=\frac{1}{2} \iint_{0}^{\infty}\left\{\left[(u+v)^{k}-u^{k}-v^{k}\right]\right. \\
&\left.\times \beta(u, v) N_{i}(u) N_{i}(v)\right\} d u d v, \\
& D_{k}^{i j}=-\iint_{0}^{\infty} u^{k} \beta(u, v) N_{i}(u) N_{j}(v) d u d v, \\
& E_{k}^{i j}=\iint_{0}^{\infty}\left\{\left[(u+v)^{k}-v^{k}\right]\right. \\
&\left.\quad \times \beta(u, v) N_{i}(u) N_{j}(v)\right\} d u d v .
\end{aligned}
$$

Note that $C_{k}^{i i}$ and $C_{k}^{j j}$ are only related to $N_{i}$ or $N_{j}$. These two terms represent the internal coagulation effect in the cluster $i$ or $j$. As a result, the single point binary Taylor expansion is used to deal with these two terms (at $u_{1}$ or $u_{2}$ ). The results from the typical TEMOM can be directly used. In (9) $m_{k}$ represents $k$ th moment of PSD $N_{i}$ or $N_{j}$. Consider

$$
\begin{gathered}
C_{0}^{i i}=\frac{B m_{0}^{i 2}}{81 m_{1}^{i 2}}\left(-151 m_{1}^{i 4}-2 m_{2}^{i 2} m_{0}^{i 2}-13 m_{2}^{i} m_{1}^{i 2} m_{0}^{i}\right), \\
C_{1}^{i i}=0, \\
C_{2}^{i i}=\frac{-2 B_{1}}{81 m_{1}^{i 2}}\left(2 m_{2}^{i 2}-13 m_{2}^{i} m_{1}^{i 2} m_{0}^{i}-151 m_{1}^{i 4}\right) .
\end{gathered}
$$

The approximation of $D_{k}^{i j}$ and $E_{k}^{i j}$ will be deduced in the following part. Substitute (4) into (7) and (8). A lot of fractional moments will appear in the expression of $D_{k}^{i j}$ and $E_{k}^{i j}$, which can be approximated through the Taylor expansion of $v^{p}$ ( $p$ is fraction) at $u_{1}$ or $u_{2}$. Consider

$$
\begin{aligned}
m_{p} \approx & \frac{u^{p-2}\left(p^{2}-p\right)}{2} m_{2} \\
& -u^{p-1}\left(p^{2}-2 p\right) m_{1}+\frac{u^{p}}{2}\left(p^{2}-3 p+2\right) m_{0} .
\end{aligned}
$$

Making use of (10), $D_{k}^{i j}$ and $E_{k}^{i j}$ can be expressed as a linear combination of $m_{k}^{i}$ and $m_{k}^{j}$. Moreover

$$
\begin{aligned}
D_{0} & =-\frac{\sum a_{m n} m_{m}^{i} m_{n}^{j}}{81}, & D_{1} & =\frac{\sum b_{m n} m_{m}^{i} m_{n}^{j}}{81}, \\
D_{2} & =\frac{\sum c_{m n} m_{m}^{i} m_{n}^{j}}{81}, & E_{0} & =0, \\
E_{1} & =\frac{\sum d_{m n} m_{m}^{i} m_{n}^{j}}{81}, & E_{2} & =-\frac{\sum e_{m n} m_{m}^{i} m_{n}^{j}}{81} .
\end{aligned}
$$

The exact expressions of the coefficients in $D_{0}, D_{1}, D_{2}, E_{1}$, and $E_{2}$ are listed in the appendix.

\section{Tests and Discussion}

In order to verify the deduction, both theoretical and numerical validations are performed, respectively.

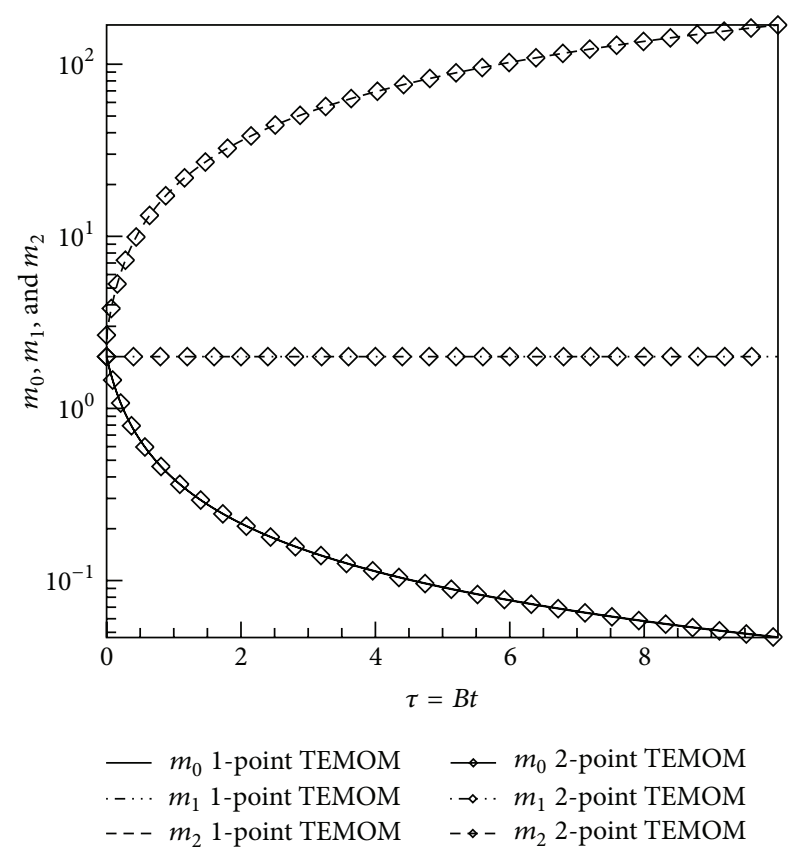

Figure 1: The evolution of moments for Case I using different expansion schemes.

Note that, if $N_{i}=N_{j}=N / 2$, (5) turns into two sets of moment equations with monomodal distribution. If (5) and set $m_{k}=m_{k}^{i}+m_{k}^{j}$, the theoretical systematic moment equations are attained:

$$
\frac{\partial m_{k}}{\partial t}=4 C_{k}^{i i}
$$

Substitute $D_{0}, D_{1}, D_{2}, E_{1}$, and $E_{2}$ into (5), and set $u_{1}=$ $u_{2}=m_{1} / m_{0}, r=1$. The right side of new equation just equals 4 times of (9), which is consistent with the theoretical equation (12).

Two simple bimodal systems are simulated to validate the current model. The single point and multipoint expansion methods are both taken into account and the results are compared with each other to show the validity and accuracy. The initial size distributions both satisfy the log-normal distribution as follows:

$$
N(v, t)=N_{0} \exp \frac{\left(-\left(\ln ^{2}\left(v / v_{g}\right)\right) /\left(2 w_{g}^{2}\right)\right)}{\left(\sqrt{2 \pi} v w_{g}\right)} .
$$

For Case I, $N_{0}^{i}=N_{0}^{j}=1.0, v_{g}^{i}=v_{g}^{j}=\sqrt{3} / 2$, and $w_{g}^{i}=$ $w_{g}^{j}=\sqrt{\ln (4 / 3)}[8]$, which represents a monomodal system and the PSD is separated into two equal sub-PSDs. For Case II, $N_{0}^{i}=1.0, v_{g}^{i}=\sqrt{3} / 2$ and $w_{g}^{i}=\sqrt{\ln (4 / 3)}$ and $N_{0}^{j}=0.1 N_{0}^{i}$, $v_{g}^{j}=1000 v_{g}^{i}$, and $w_{g}^{j}=0.1 w_{g}^{i}$, which represents a bimodal system consisting of two log-normal sub-PSDs.

Figure 1 shows the results of Case I for both single point TEMOM and multipoint TEMOM. From the figure, a good agreement is obtained. This is because the particle system is, in the final analysis, a mono-modal system. The consistency 


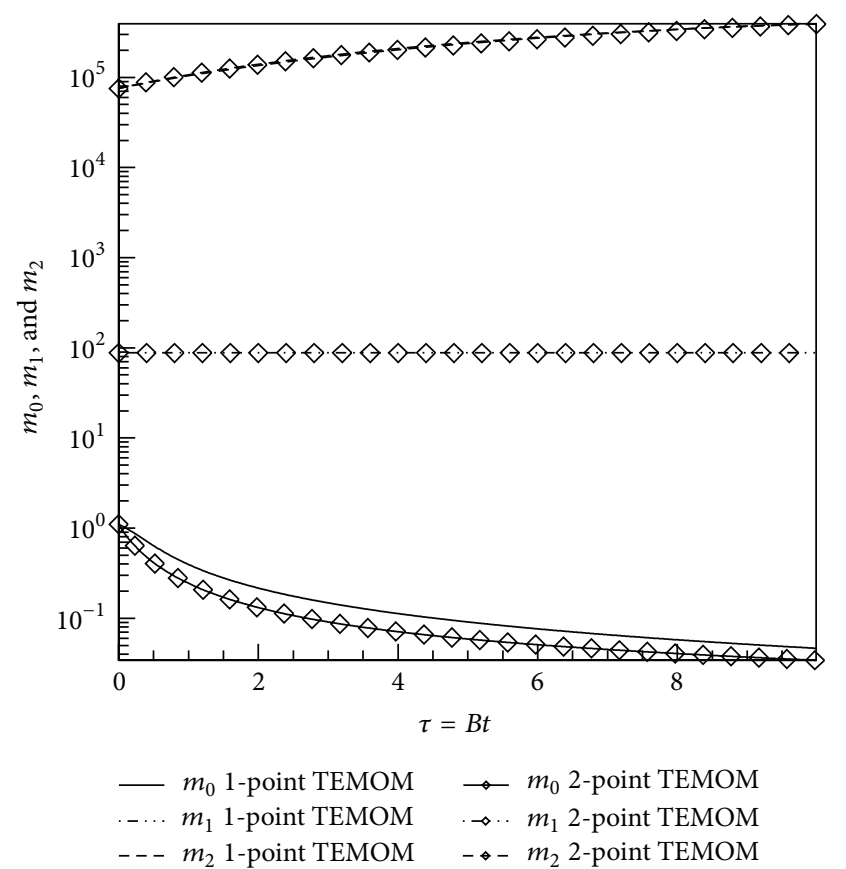

Figure 2: The evolution of moments for Case II using different expansion schemes.

between two methods is just as the same as the theoretical analysis at the beginning of this paragraph.

Figure 2 shows the results of Case II for both single point TEMOM and multi point TEMOM. From the figure, an obvious deviation is found. It shows that, for a typical bimodal system, the particle size difference between different models can not be neglected. The value for multipoint TEMOM is always smaller than that for TEMOM especially for $m_{0}$. This means that the original TEMOM model will underestimate the coagulation effect for the particle number concentration $\left(m_{0}\right)$. Another interesting phenomenon is that $m_{1}$ is the same for both of the two models. The reason is that $m_{1}$ physically represents the volume fraction of particles. The particle collision (coagulation) will not change the total volume or the mass of particles. Hence, $m_{1}$ is a constant from the beginning to the end.

Define the error function as

$$
E_{k}=\frac{m_{k}^{\mathrm{m}}-m_{k}^{\mathrm{s}}}{m_{k}^{\mathrm{s}}}
$$

Where $m_{k}^{\mathrm{m}}$ represents the moments in multi-point TEMOM and $m_{k}^{s}$ represents the moments in original TEMOM. The exact tendency of $E_{k}$ is shown in Figure 3. According to the figure, the maximum deviation for $m_{0}$ will be about $15 \%$ and $4 \%$ for $m_{2}$. For $m_{0}$, the error function $E_{0}$ will increase in a very short time, reach the maximum, and then decrease slowly. This phenomenon indicates that the difference in particle size will lead to a relatively large deviation at the very beginning of coagulation for bimodal particle system when the TEMOM is selected for the bimodal particle system.

Figure 4 shows the different moments in modes $i$ and $j$ using the technique proposed in current paper. According to

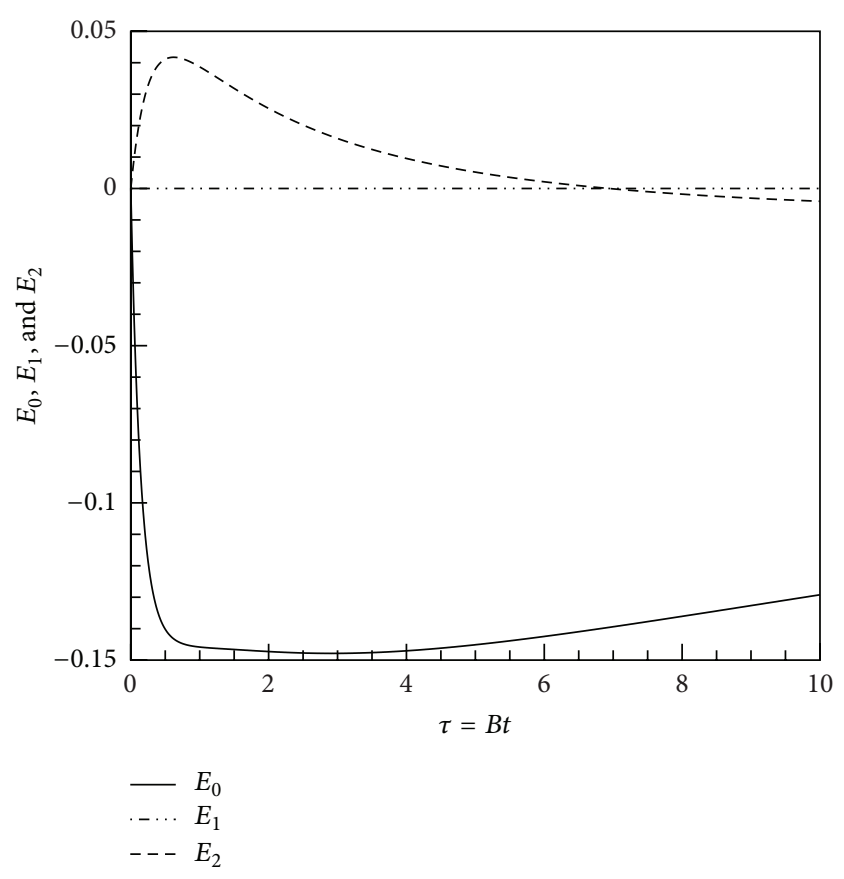

FIGURE 3: The variation of error function $E_{k}$ versus dimensionless time $\tau$.

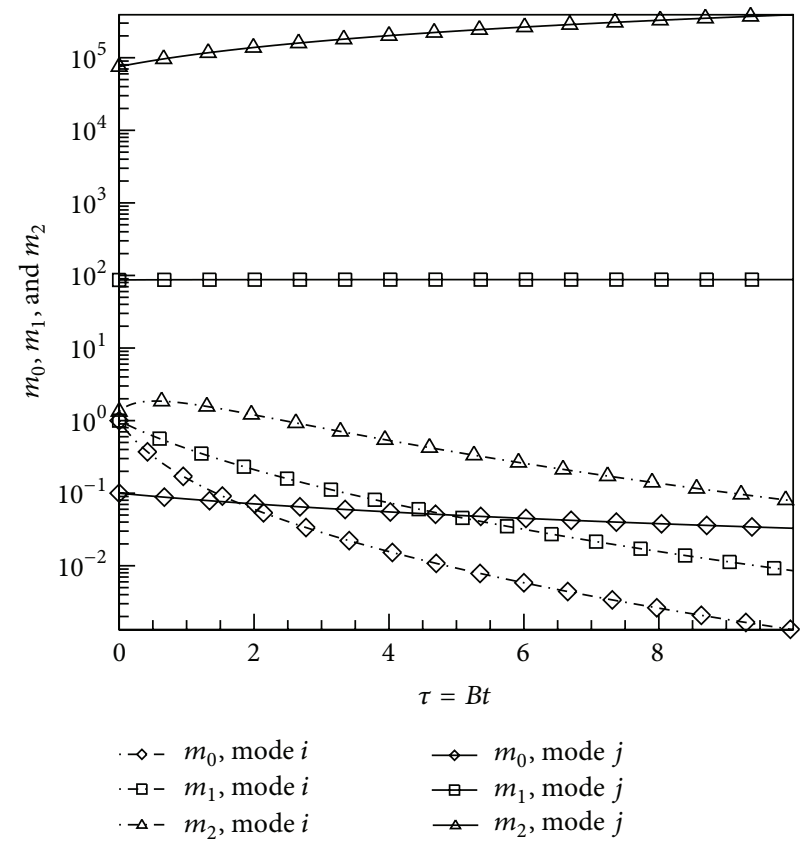

Figure 4: The evolution of moments for Case II with different modes.

the figure, an obvious reduction is found for each moment $m_{k}$ in mode $i$, which means that the coagulation will lead to the decrease of $m_{0}$ (particle number concentration) and $m_{1}$ (particle volume fraction). Particularly the volume fraction of particles, $m_{1}$, no longer keeps a constant because of the external collision with particles in mode $j$ and the new birth of bigger particles. For particles in mode $j$, the internal 
coagulation in mode $j$ will lead to the decrease of $m_{0}$, while the external coagulation between mode $i$ and mode $j$ will take no effect on $m_{0}$. As a result, the slope of curve is flatter than that in Figure 2. However, $m_{1}$ and $m_{2}$ are comparable with those in Figure 2, because these two parameters are related to the particle volume tightly. The average volume of particle in mode $j$ is much bigger than that in mode $i$, according to the initial condition. In general, such a result indicates the importance of current technique, giving more accurate result and more detail for the complex bimodal particle system.

\section{Conclusions}

The current research showed a multipoint Taylor-expansion method of moments for the bimodal particle system in the Stokes regime. A theoretical deduction was performed and brief results are given. Both theoretical validation and numerical tests are implemented. The results show that, for a single-modal system, there is no difference between the two methods. However, for a bimodal system, although the evolution of moments has the same tendency, there is obvious deviation between the two methods. For the case investigated in current paper, the maximum deviation for $m_{0}$ is about $15 \%$ and $4 \%$ for $m_{2}$. Each moment $m_{k}$ in mode $i$ will decrease. The technique proposed in this paper will bring in the accuracy and details of particles. This method can be further extended to the multi-modal system

\section{Appendix}

The coefficients in (11) are listed with the definition $r=$ $\left(u_{1} / u_{2}\right)^{1 / 3}$. Consider

$$
\begin{array}{cc}
a_{00}=70 r+70 r^{-1}+162, & a_{01}=35 u_{1}^{-1}\left(2 r^{2}-r^{4}\right), \\
a_{02}=u_{1}^{-2}\left(10 r^{7}-14 r^{5}\right), & a_{10}=35 u_{1}^{-1}\left(2 r-r^{-1}\right), \\
a_{11}=-35 u_{1}^{-2}\left(r^{4}+r^{2}\right), & a_{12}=u_{1}^{-3}\left(10 r^{7}+7 r^{5}\right), \\
a_{20}=u_{1}^{-2}\left(10 r^{-1}-14 r\right), & a_{21}=u_{1}^{-3}\left(7 r^{4}+10 r^{2}\right), \\
a_{22}=-2 u_{1}^{-4}\left(r^{7}+r^{5}\right) ; & b_{01}=-7 r^{4}-10 r^{2}, \\
b_{02}=2 u_{1}^{-1}\left(r^{7}+r^{5}\right), \quad b_{10}=-112 r-40 r^{-1}-162, & b_{12}=8 u_{1}^{-2}\left(r^{5}-2 r^{7}\right), \\
b_{11}=u_{1}^{-1}\left(56 r^{4}-40 r^{2}\right), & b_{21}=u_{1}^{-2}\left(14 r^{4}+5 r^{2}\right), \\
b_{20}=u_{1}^{-1}\left(5 r^{-1}-28 r\right), & b_{22}=-u_{1}^{-3}\left(4 r^{7}+r^{5}\right) ; \\
c_{00}=u_{1}^{2}\left(5 r^{-1}-28 r\right), & c_{01}=u_{1}\left(14 r^{4}+5 r^{2}\right), \\
c_{02}=-4 r^{7}-r^{5}, \quad c_{10}=u_{1}\left(98 r-25 r^{-1}\right),
\end{array}
$$

$$
\begin{gathered}
c_{11}=-49 r^{4}-25 r^{2}, \quad c_{12}=u_{1}^{-1}\left(14 r^{7}+5 r^{5}\right) \\
c_{20}=-196 r-25 r^{-1}-162, \quad c_{21}=u_{1}^{-1}\left(98 r^{4}-25 r^{2}\right), \\
c_{22}=u_{1}^{-2}\left(5 r^{5}-28 r^{7}\right) ; \\
d_{00}=u_{1}\left(10 r^{-1}-14 r\right), \quad d_{01}=7 r^{4}+10 r^{2} \\
d_{02}=-2 u_{1}^{-1}\left(r^{7}+r^{5}\right), \quad d_{10}=112 r+40 r^{-1}+162 \\
d_{11}=u_{1}^{-1}\left(40 r^{2}-56 r^{4}\right), \quad d_{12}=8 u_{1}^{-2}\left(2 r^{7}-r^{5}\right) \\
d_{20}=u_{1}^{-1}\left(28 r-5 r^{-1}\right), \quad d_{21}=-u_{1}^{-2}\left(14 r^{4}+5 r^{2}\right) \\
d_{22}=u_{1}^{-3}\left(4 r^{7}+r^{5}\right) ; \\
e_{00}=u_{1}^{2}\left(-28 r+5 r^{-1}+4 r^{-2}+4 r^{-4}\right) \\
e_{01}=u_{1}\left(14 r^{4}+5 r^{2}+16 r-32 r^{-1}\right) \\
e_{21}=u_{1}^{-1}\left(98 r^{4}-25 r^{2}-32 r+16 r^{-1}\right) \\
e_{02}=-4 r^{7}-r^{5}-2 r^{4}-8 r^{2} \\
e_{10}= \\
e_{11}=-49 r^{4}-25 r^{2}-128 r-128 r^{-1}-324 \\
\left(98 r-25 r^{-1}-32 r^{-2}+16 r^{-4}\right) \\
5
\end{gathered}
$$

\section{Conflict of Interests}

The authors declare that there is no conflict of interests regarding the publication of this paper.

\section{Acknowledgments}

The authors gratefully acknowledges the financial support from the National Natural Science Foundation of China under Grant no. 11302070, the National Basic Research Program of China (973 Program) under Grant no. 2010CB227102 .

\section{References}

[1] T. L. Chan, Z. Ning, J. S. Wang, C. S. Cheung, C. W. Leung, and W. T. Hung, "Gaseous and particle emission factors from the selected on-road petrol/gasoline, diesel, and liquefied petroleum gas vehicles," Energy and Fuels, vol. 21, no. 5, pp. 2710-2718, 2007.

[2] R. B. Diemer and J. H. Olson, "A moment methodology for coagulation and breakage problems, part 2: moment models and distribution reconstruction," Chemical Engineering Science, vol. 57, no. 12, pp. 2211-2228, 2002. 
[3] J. I. Jeong and M. Choi, "A bimodal moment model for the simulation of particle growth," Journal of Aerosol Science, vol. 35, no. 9, pp. 1071-1090, 2004.

[4] A. Pugatshova, A. Reinart, and E. Tamm, "Features of the multimodal aerosol size distribution depending on the air mass origin in the Baltic region," Atmospheric Environment, vol. 41, no. 21, pp. 4408-4422, 2007.

[5] G. Lonati, M. Crippa, V. Gianelle, and R. van Dingenen, "Daily patterns of the multi-modal structure of the particle number size distribution in Milan, Italy," Atmospheric Environment, vol. 45, no. 14, pp. 2434-2442, 2011.

[6] H. Tang and J. Lin, "Research on bimodal particle extinction coefficient during Brownian coagulation and condensation for the entire particle size regime," Journal of Nanoparticle Research, vol. 13, no. 12, pp. 7229-7245, 2011.

[7] A. S. Koziol and H. G. Leighton, "The moments method for multi-modal multi-component aerosols as applied to the coagulation-type equation," Quarterly Journal of the Royal Meteorological Society, vol. 133, no. 625, pp. 1057-1070, 2007.

[8] J. C. Barrett and J. S. Jheeta, "Improving the accuracy of the moments method for solving the aerosol general dynamic equation," Journal of Aerosol Science, vol. 27, no. 8, pp.1135-1142, 1996.

[9] C. H. Jung and Y. P. Kim, "Numerical estimation of the effects of condensation and coagulation on visibility using the moment method," Journal of Aerosol Science, vol. 37, no. 2, pp. 143-161, 2006.

[10] R. McGraw, "Description of aerosol dynamics by the quadrature method of moments," Aerosol Science and Technology, vol. 27, no. 2, pp. 255-265, 1997.

[11] M. Yu, J. Lin, and T. Chan, "A new moment method for solving the coagulation equation for particles in Brownian motion," Aerosol Science and Technology, vol. 42, no. 9, pp. 705-713, 2008.

[12] M. Yu and J. Lin, "Taylor-expansion moment method for agglomerate coagulation due to Brownian motion in the entire size regime," Journal of Aerosol Science, vol. 40, no. 6, pp. 549562,2009 .

[13] M. Yu and J. Lin, "Binary homogeneous nucleation and growth of water-sulfuric acid nanoparticles using a TEMOM model," International Journal of Heat and Mass Transfer, vol. 53, no. 4, pp. 635-644, 2010.

[14] M. Yu, J. Lin, and T. Chan, "Numerical simulation for nucleated vehicle exhaust particulate matters via the temom/les method," International Journal of Modern Physics C, vol. 20, no. 3, pp. 399421, 2009.

[15] J. Lin, P. Lin, and H. Chen, "Research on the transport and deposition of nanoparticles in a rotating curved pipe," Physics of Fluids, vol. 21, no. 12, pp. 1-11, 2009.

[16] M. Yu, J. Lin, H. Jin, and Y. Jiang, "The verification of the Taylorexpansion moment method for the nanoparticle coagulation in the entire size regime due to Brownian motion," Journal of Nanoparticle Research, vol. 13, no. 5, pp. 2007-2020, 2011. 


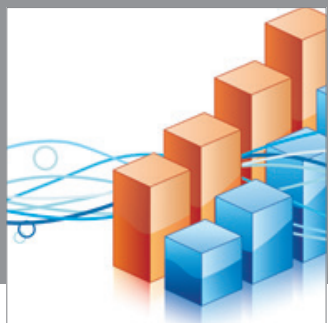

Advances in

Operations Research

mansans

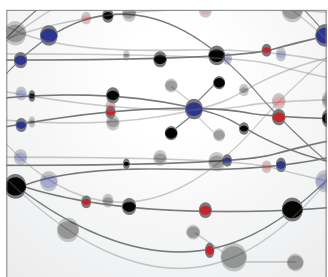

The Scientific World Journal
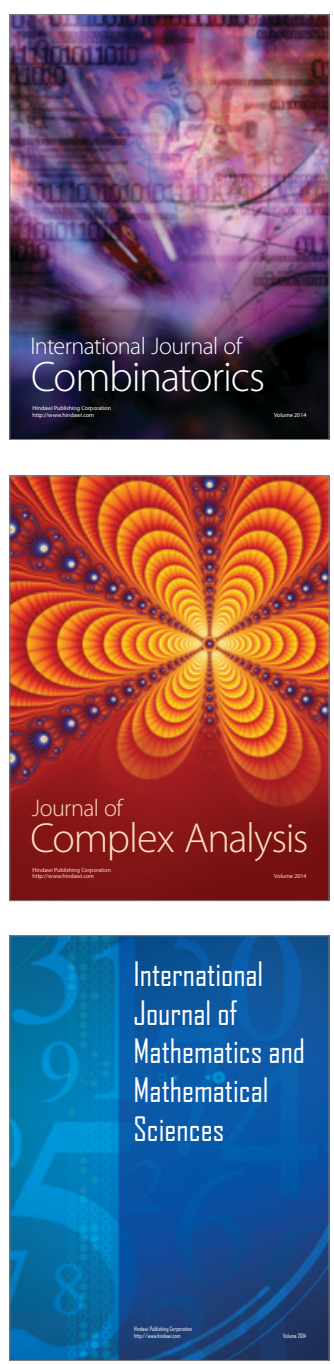
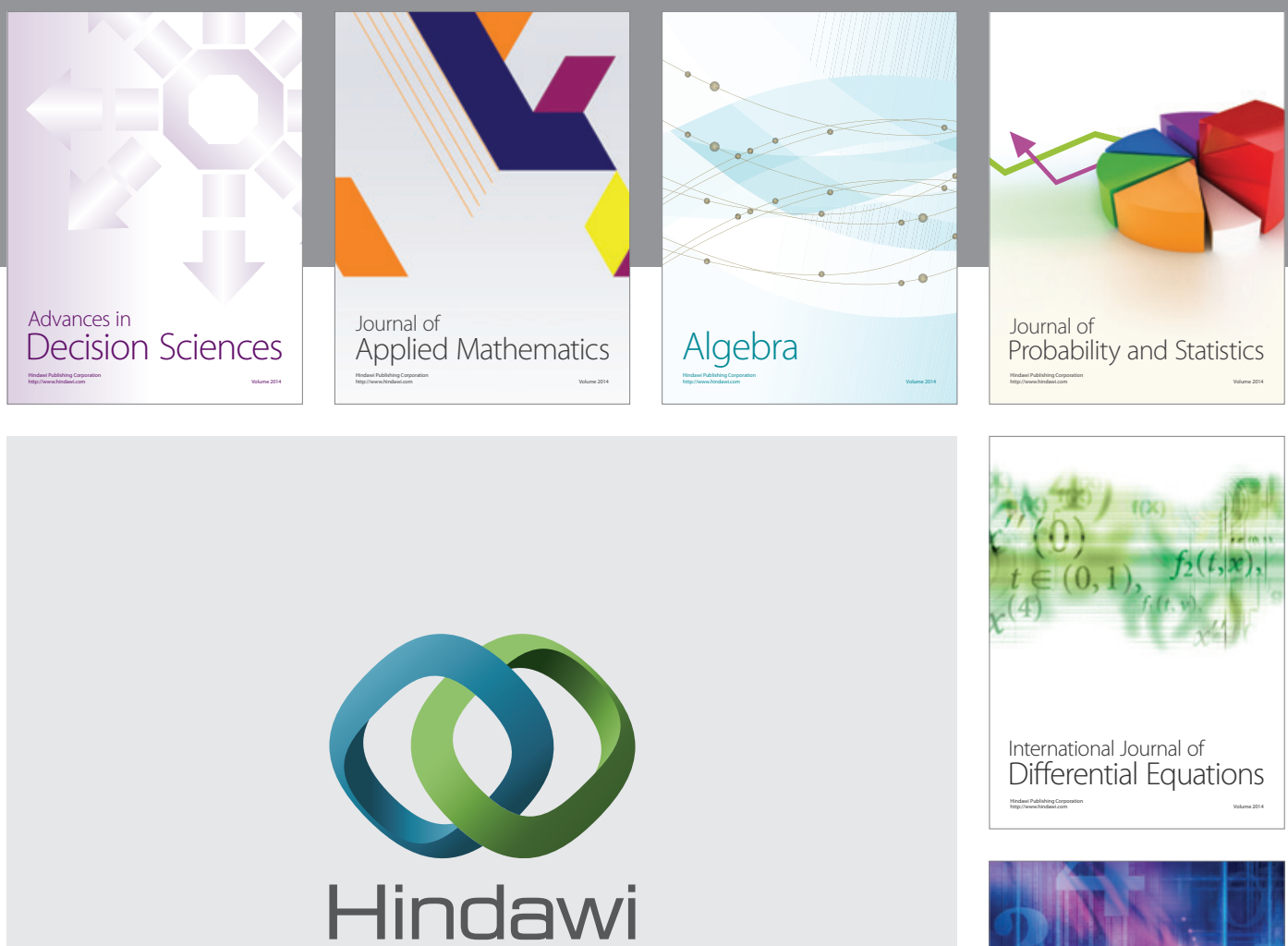

Submit your manuscripts at http://www.hindawi.com
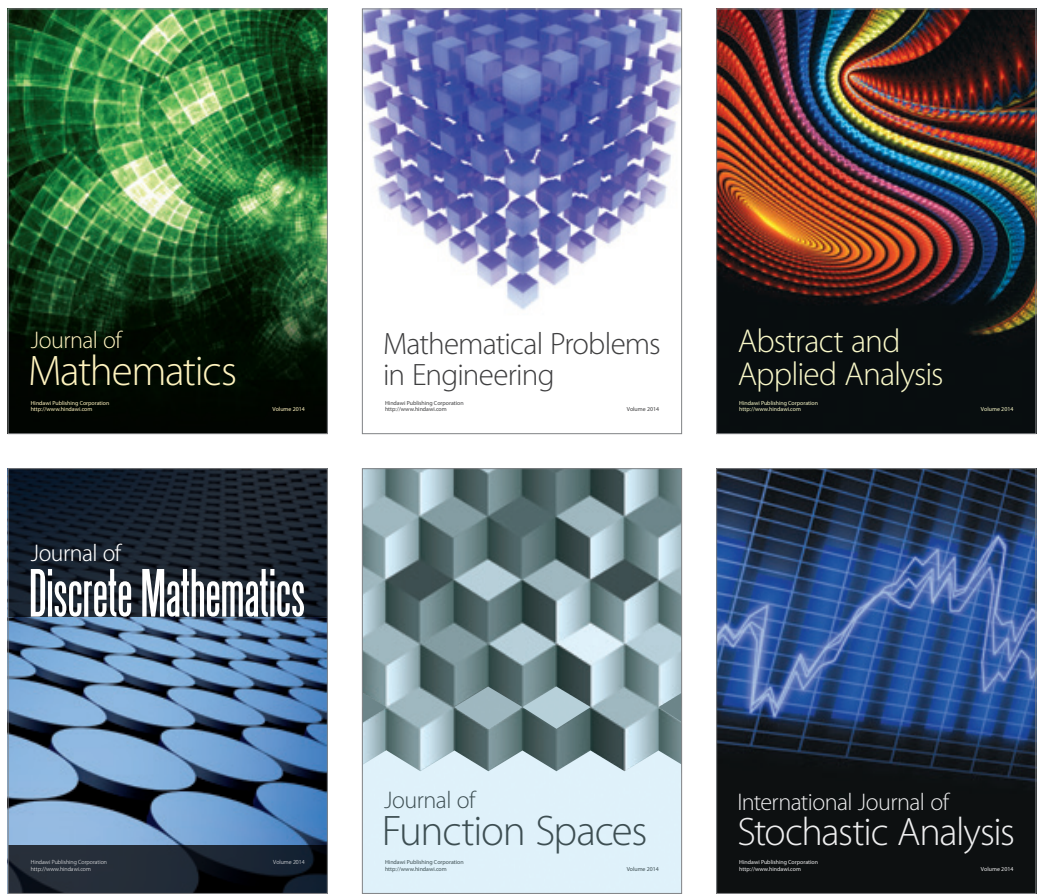

Journal of

Function Spaces

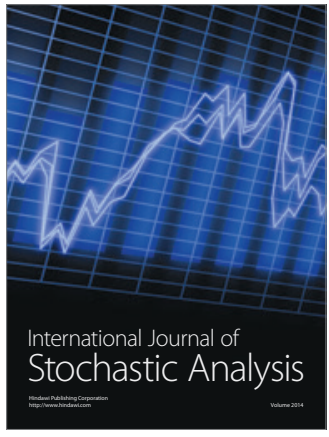

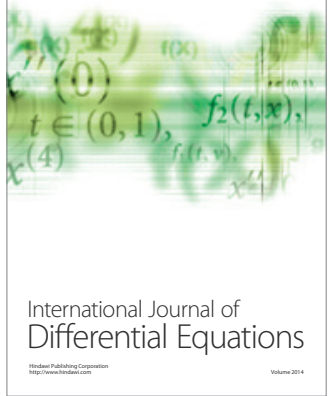
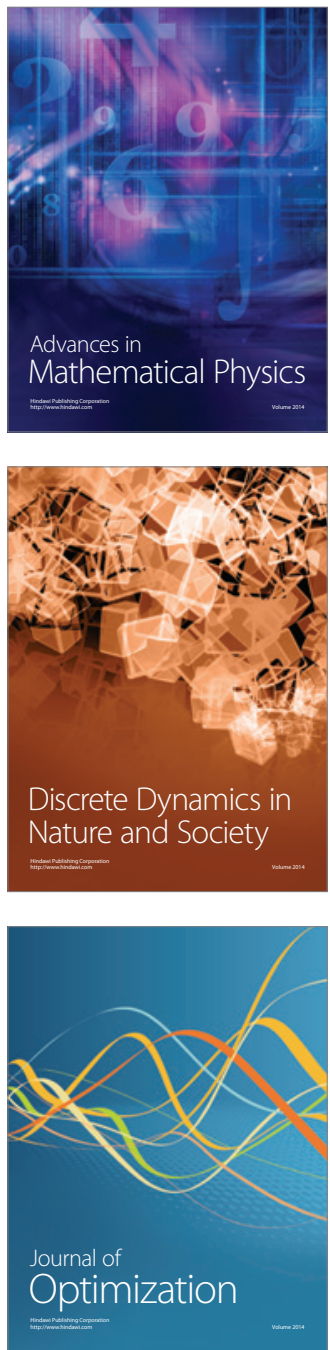